\title{
Spin-density induced by electromagnetic wave in two-dimensional electron gas
}

\author{
Alexander Shnirman $^{1}$ And Ivar Martin ${ }^{2}$ \\ Institut für Theoretische Festkörperphysik, Universität Karlsruhe, D-76128 Karlsruhe, Germany. \\ Theoretical Division, Los Alamos National Laboratory, Los Alamos, NM 87545, USA.
}

PACS 72.25.Rb - Spin relaxation and scattering

PACS 85.75.-d - Magnetoelectronics; spintronics: devices exploiting spin polarized transport or integrated magnetic fields

\begin{abstract}
We consider the magnetic response of a two-dimensional electron gas (2DEG) with a spin-orbit interaction to a long-wave-length electromagnetic excitation. We observe that the transverse electric field creates spin polarization perpendicular to the 2DEG plane. The effect is more prominent in clean systems with resolved spin-orbit-split subbands, and reaches maximum when the frequency of the wave matches the subband splitting at the Fermi momentum. The relation of this effect to the spin-Hall effect is discussed.
\end{abstract}

Spintronics is a fast developing field of research. While the spin-orbit effects in semiconductors have been discussed for a long time [1-3], recently discovered spin-Hall effect $[4,5]$ has generated a lot of interest in applying spinorbit related effects to spintronics [6]. The promise of the spin-Hall effect is in the possibility of generation of non-equilibrium spin polarization by means of a DC electric field. However, after some discussions it was conGcluded that in two-dimensional electron gases with the I Rashba and the Dresselhaus spin-orbit interactions, the spin-Hall effect vanishes for constant and homogeneous electric field [7-10]. At finite frequencies the spin-Hall effect is non-zero [8].

While for the homogeneous spin-Hall effect (uniform applied electric field) the out-of-plane spin polarization is expected to accumulate only at the edges of the sample [8], there is an alternative possibility that we propose to explore here: To create an out-of-plane inhomogeneous spin density in the bulk in response to a spatially-modulated field. Such bulk accumulation is free from the uncertainties associated with the charge and spin transport near the sample boundaries, and thus may provide an unambiguous method to detect the spin-Hall effect.

A possible realization is shown in Fig. 1, and corresponds to a transverse electric field $E_{x}$ changing in the $y$-direction. In the presence of the spin-Hall effect, the spin currents are expected to flow towards each other, with the spin density accumulating in the middle. From the Maxwell equation, $\operatorname{rot} \boldsymbol{E}=-(1 / c) \dot{B}$, a

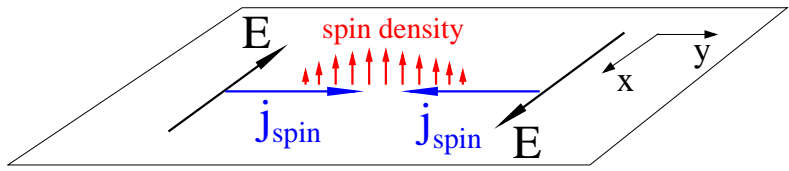

Fig. 1: The "naive" motivation: Spin accumulation due to converging spin currents.

transverse electric field can only exist together with the time-dependent magnetic field. To create the situation shown in Fig. 1 we therefore consider a linearly polarized in-plane microwave field $\boldsymbol{A}=\boldsymbol{e}_{x} A_{0} \exp \left(i q_{y} y-i \Omega t\right)$; $\boldsymbol{E}=E_{x} \boldsymbol{e}_{x}=\boldsymbol{e}_{x}\left(A_{0} / c\right) i \Omega \exp \left(i q_{y} y-i \Omega t\right) ; \boldsymbol{B}=B_{z} \boldsymbol{e}_{z}=$ $-\boldsymbol{e}_{z} A_{0} i q_{y} \exp \left(i q_{y} y-i \Omega t\right)$; and $\Omega=c|\boldsymbol{q}|=c q_{y}$. There is naturally a spin response in the $z$-direction due to the $B_{z}$ magnetic field (the Zeeman effect; this is similar to the response to an in-plane field considered, e.g., in Refs. [11,12]). However, in addition to this pure spin effect, we also find an orbital contribution, $s_{z}^{\text {orbital }} \sim \operatorname{rot} \boldsymbol{E}$, which can be attributed to the spin-Hall effect. (Since $\operatorname{rot} \boldsymbol{E} \sim \Omega B_{z}$ this response can be conveniently interpreted as an orbital contribution to the spin susceptibility.) The response of the in-plane polarization to the transverse electric field at arbitrary values of $\boldsymbol{q}$ was considered for the clean case in Ref. [13].

We consider the Rashba model of a 2DEG with the 
Hamiltonian

$H=\frac{\left(\boldsymbol{p}-\frac{e}{c} \boldsymbol{A}\right)^{2}}{2 m^{*}}+\alpha_{\mathrm{R}} \boldsymbol{\eta}\left(\boldsymbol{p}-\frac{e}{c} \boldsymbol{A}\right)-\frac{1}{2} g \mu_{\mathrm{B}} \boldsymbol{B} \boldsymbol{\sigma}+V_{\text {disorder }}$.

where $\boldsymbol{\eta} \equiv \boldsymbol{z} \times \boldsymbol{\sigma}=\left(-\sigma_{y}, \sigma_{x}, 0\right)$ and $m^{*}$ is the effective electron band mass. The model (with $\boldsymbol{A}=0$ and $V_{\text {disorder }}=0$ ) has two energy bands. The eigenfunctions of the two bands $\beta= \pm$ are given by

$$
\left|u_{ \pm, p}\right\rangle=\frac{1}{\sqrt{2}}\left(|\uparrow\rangle \mp i e^{i \phi_{p}}|\downarrow\rangle\right)
$$

where $\tan \phi_{p} \equiv p_{y} / p_{x}$ and the energies are given by $\epsilon^{ \pm}(\boldsymbol{p})=\frac{\boldsymbol{p}^{2}}{2 m^{*}} \pm \Delta_{p}$, where $\Delta_{p} \equiv \alpha_{\mathrm{R}}|\boldsymbol{p}|$.

One should distinguish between the clean and dirty regimes. a) In the clean regime (weak disorder or strong SOI) $\Delta_{F} \equiv \alpha_{\mathrm{R}} p_{F} \gg \tau^{-1}$, where $\tau$ is the elastic mean free time. The two spin-orbit split bands are, thus, well defined. b) In the dirty regime (strong disorder or weak SOI) $\Delta_{F} \ll \tau^{-1}$. The band splitting is not resolved. This is the regime where the Dyakonov-Perel relaxation applies [1]. One can also define a "super clean" regime $1 / \tau<m^{*} \alpha_{\mathrm{R}}^{2}$, which, however, appears to be irrelevant experimentally at this time and will not be considered here.

In this work we focus primarily on the clean regime, although our diagrammatic derivation holds also in the dirty regime. Depending on the frequency of the applied field one can then probe the resonant $\left(\Omega \approx 2 \Delta_{F}\right)$ and offresonant $\left(\left|\Omega-2 \Delta_{F}\right| \gg 1 / \tau\right.$, e.g. $\left.\Omega \ll 2 \Delta_{F}\right)$ responses of the spin system. Before rigorously deriving our results diagrammatically, we provide qualitative derivation of spin responses in these regimes.

In the low-frequency (off-resonant) limit, we consider slow (both in time and space) components of the singleelectron density matrix $f_{\beta_{1}, \beta_{2}}\left(\boldsymbol{p}_{1}, \boldsymbol{p}_{2}\right)$. Here $\beta_{1}$ and $\beta_{2}$ are the band indexes while $\boldsymbol{p}_{1}$ and $\boldsymbol{p}_{2}$ are the electron momenta. Introducing $\boldsymbol{p}=\left(\boldsymbol{p}_{1}+\boldsymbol{p}_{2}\right) / 2$ and $\boldsymbol{q}=\boldsymbol{p}_{1}-\boldsymbol{p}_{2}$ we note that slowness in space means that $|\boldsymbol{q}|$ is small. When the spin-orbit splitting, $\Delta_{p}$, is large, only the banddiagonal elements $f_{\beta}\left(\boldsymbol{p}_{1}, \boldsymbol{p}_{2}\right) \equiv f_{\beta, \beta}\left(\boldsymbol{p}_{1}, \boldsymbol{p}_{2}\right)$ can be slow. Indeed the band-off-diagonal elements either oscillate in time with frequency $\sim \Delta_{p}$ or oscillate in space with $|\boldsymbol{q}| \sim$ $\Delta_{p} / v_{F}$. Consider the density matrix of band $\beta$ in the coordinate representation:

$$
\begin{aligned}
& f_{\beta}\left(\boldsymbol{r}, \boldsymbol{r}^{\prime}\right)= \\
& \sum_{\boldsymbol{p}_{1}, \boldsymbol{p}_{2}} f_{\beta}\left(\boldsymbol{p}_{1}, \boldsymbol{p}_{2}\right) e^{\left(i \boldsymbol{p}_{1} \boldsymbol{r}_{-i} \boldsymbol{p}_{2} \boldsymbol{r}^{\prime}\right)}\left|u_{\left.\beta, \boldsymbol{p}_{1}\right\rangle\left\langle u_{\beta}, \boldsymbol{p}_{2}\right|}\right|
\end{aligned}
$$

We calculate the $z$-component of the spin density (cf. Ref. [14]):

$$
\begin{aligned}
s_{z}(\boldsymbol{r}) & =\frac{1}{2} \sum_{\beta} \operatorname{Tr}\left[\sigma_{z} f_{\beta}(\boldsymbol{r}, \boldsymbol{r})\right] \\
& \approx \frac{1}{2} \sum_{\beta, \boldsymbol{p}, \boldsymbol{q}} f_{\boldsymbol{p}, \boldsymbol{q}, \beta} e^{i \boldsymbol{q} \boldsymbol{r}}\left\langle u_{\beta, \boldsymbol{p}}\left|\sigma_{z} \boldsymbol{q} \cdot \nabla \boldsymbol{p}\right| u_{\beta, \boldsymbol{p}}\right\rangle
\end{aligned}
$$

$$
=\frac{1}{2} \sum_{\beta, \boldsymbol{p}, \boldsymbol{q}} f_{\boldsymbol{p}, \boldsymbol{q}, \beta} e^{i \boldsymbol{q} \boldsymbol{r}}\left(-i \boldsymbol{q} \cdot \nabla \boldsymbol{p} \phi_{p} / 2\right)
$$

Note that the contributions of both bands add up. For $\boldsymbol{q}=q_{y} \boldsymbol{e}_{\boldsymbol{y}}$ and using $\nabla_{p_{y}} \phi_{p}=\cos \phi_{p} / p$ we obtain $s_{z}(\boldsymbol{q}) \propto i q_{y} \sum_{\beta, \boldsymbol{p}} f_{\boldsymbol{p}, \boldsymbol{q}, \beta} \cos \phi_{p} / p$. From the usual Boltzmann equation we know that under the influence of the electric field $\boldsymbol{E}=E_{x} \boldsymbol{e}_{x}$ the correction to the distribution function satisfies $\sum_{\boldsymbol{p}} f_{\boldsymbol{p}, \boldsymbol{q}, \beta} \cos \phi_{p} \propto \nu e E_{x} v_{F} \tau$, where $\nu$ is the density of states and $\tau$ is the momentum relaxation time. Thus we finally obtain $s_{z}(\boldsymbol{q}) \propto i q_{y} e E_{x} \tau$ (we have used $p_{F}=m^{*} v_{F}, \nu \propto m^{*}$ and the fact that everything happens near the Fermi energy).

When the excitation frequency approximately matches the spin subband splitting (the resonant case), the generation of the transverse spin polarization can be understood based on the Bloch equations, familiar from the theory of the nuclear and electron spin resonance [15]. The spinorbit coupling can be represented as a momentum dependent magnetic field $\boldsymbol{b}_{0}$ acting on the electrons with the Hamiltonian $H_{S O}=\boldsymbol{b}_{0} \boldsymbol{\sigma}$, where $\boldsymbol{b}_{0}=\Delta_{p}\left(p_{y} / p,-p_{x} / p\right)$. The spin-dependent part of the interaction of the EM wave with electrons can be recast in the following form, $H_{\text {int }}=\boldsymbol{b}_{1} \boldsymbol{\sigma}$ where $\boldsymbol{b}_{1}$ is a small oscillating magnetic field directed along the $y$-axis, $\boldsymbol{b}_{1}=\alpha_{\mathrm{R}}(e / c) A_{0} \boldsymbol{e}_{y} e^{i q_{y} y-i \Omega t}$. The single-electron states $\left|u_{-, \boldsymbol{p}}\right\rangle$ and $\left|u_{+, \boldsymbol{p}+\boldsymbol{q}\rangle}\right\rangle$ that are connected by the AC field $\boldsymbol{b}_{1}$ do not have the same momentum, and thus their energy difference is given by $\delta E_{p}=\epsilon^{+}(\mathbf{p}+\mathbf{q})-\epsilon^{-}(\mathbf{p}) \approx 2 \Delta_{p}+p_{y} q_{y} / m^{*}$. For each such pair of states, provided that, without the driving, $\left|u_{-}, \boldsymbol{p}\right\rangle$ is occupied while $\left|u_{+, \boldsymbol{p}+\boldsymbol{q}}\right\rangle$ is not, the usual Bloch dynamics of the electron spin takes place. That is $\left|u_{-,}, \boldsymbol{p}\right\rangle$ plays the role of the state $|\uparrow\rangle$ along the direction of $\boldsymbol{b}_{0}$, while $\left|u_{+}, \boldsymbol{p}+\boldsymbol{q}\right\rangle$ plays the role of $|\downarrow\rangle$. Only the part of $\boldsymbol{b}_{1}$ perpendicular to $\boldsymbol{b}_{0}$ is important, $\left|\boldsymbol{b}_{1, \perp}\right|=\left|\boldsymbol{b}_{1}\right| \sin \phi_{p}$. In the linear response regime the driving produces the outof-plane polarization

$$
\left\langle\sigma_{z, \boldsymbol{p}}\right\rangle \sim \operatorname{Re}\left[\frac{i e^{-i \Omega t}\left|\boldsymbol{b}_{1}\right| \sin \phi_{p}}{\left[i / T_{2}+\left(\Omega-\delta E_{p}\right)\right]}\right],
$$

where $T_{2}$ is the dephasing time. $T_{2} \sim \tau$ in the clean limit. The total spin polarization is given by $s_{z}=$ $L^{-2} \sum_{\mathbf{p}}\left\langle\sigma_{z, \boldsymbol{p}}\right\rangle n_{F}\left(\epsilon_{\mathbf{p}}^{-}\right)\left[1-n_{F}\left(\epsilon_{\mathbf{p}+\mathbf{q}}^{+}\right)\right]$. The summation is effectively restricted to the ring defined by $p_{F}+\alpha_{\mathrm{R}} m^{*}>$ $p>p_{F}-\alpha_{\mathrm{R}} m^{*}$. (Note that in the limit $\boldsymbol{q}=0$ the spin polarization vanishes, since $\left\langle\sigma_{z, \boldsymbol{p}}\right\rangle \propto \sin \phi_{p}$, i.e. it has only a $p$-component. This actually means that that there is a resonant spin current $j_{y}^{z}$ flowing in the system [8].) Restricting ourselves to the experimentally relevant "notsuper-clean" regime $1 / \tau>\alpha_{\mathrm{R}}^{2} m^{*}$, all states in the ring $p_{F}+\alpha_{\mathrm{R}} m^{*}>p>p_{F}-\alpha_{\mathrm{R}} m^{*}$ are "in resonance" simultaneously (cf. (5)) when $\Omega \sim 2 \Delta_{F}$. Assuming also $v_{F}|\boldsymbol{q}|<1 / \tau$ we expand the denominator of (5) and after summation obtain

$$
s_{z} \sim \operatorname{Re}\left[\frac{i e^{-i \Omega t}(e / c) A_{0} q \Delta_{F}^{2}}{\left[i / T_{2}+\left(\Omega-2 \Delta_{F}\right)\right]^{2}}\right] .
$$


Notice the strongly resonant (Fano shape) nature of this contribution. A very similar result also obtains for the case of the Dresselhaus spin-orbit interaction. Interestingly, by noting that $A_{0} / c=E_{x} /(i \Omega)$ and replacing $\Omega \rightarrow i / \tau$ we can approximately recover the low-frequency spin accumulation from the resonant result. This indicates the close connection between these two effects.

A resonant generation of out-of-plane spin polarization was also recently proposed in Ref [16]. In that work, the polarization appeared due to explicit symmetry breaking by a strong constant magnetic field applied in the 2DEG plane, while in our case the effect is due to the finite value of $\boldsymbol{q}$.

Diagrammatic derivation. We now derive these results in a rigorous manner. We follow the route of Refs. [8, 17] and derive, first, the kinetic (diffusion) equations governing the dynamics of the charge and spin densities. We use the Keldysh technique. For introduction see Ref. [18]. Although such derivations are standard [18], for completeness, we provide here the main elements.

We consider only s-wave disorder scattering, that is $V_{\text {disorder }}=\sum_{k} u \delta\left(\boldsymbol{r}-\boldsymbol{r}_{k}\right)$ where $\boldsymbol{r}_{k}$ are random locations with the average density $n_{\mathrm{imp}}$. We employ the linear response: $H=H_{0}+H_{1}$ where

$$
H_{0}=\frac{\boldsymbol{p}^{2}}{2 m^{*}}+\alpha_{\mathrm{R}} \boldsymbol{\eta} \boldsymbol{p}+V_{\text {disorder }}
$$

and

$$
\begin{aligned}
H_{1} & =-\frac{e}{2 c}\{\boldsymbol{v}, \boldsymbol{A}\}_{+}+e \phi-\frac{1}{2} g \mu_{\mathrm{B}} \boldsymbol{B} \boldsymbol{\sigma} \\
& =-\frac{e}{2 c}\left\{\left(\frac{\boldsymbol{p}}{m^{*}}+\alpha_{\mathrm{R}} \boldsymbol{\eta}\right), \boldsymbol{A}\right\}_{+}+\hat{\Phi} .
\end{aligned}
$$

To make the calculation gauge invariant we have added a scalar potential perturbation, $\phi$. We neglect the $\boldsymbol{A}^{2}$ contribution and introduce, for convenience, a 4-potential $\hat{\Phi} \equiv e \phi \sigma_{0}-(1 / 2) g \mu_{\mathrm{B}} \boldsymbol{B} \boldsymbol{\sigma}$.

In order to account properly for the vertex corrections $[7,9]$ we perform the linear response expansion starting from the Dyson equation which reads $(i \partial / \partial t-H) * G=$ $\hat{1}+\Sigma * G$, where ${ }^{*}$ stands for convolution. The self-energy is given by the self-consistent Born approximation.

$$
\Sigma\left(\boldsymbol{r}_{1}, \boldsymbol{r}_{2}, t_{1}, t_{2}\right)=n u^{2} \delta\left(\boldsymbol{r}_{1}-\boldsymbol{r}_{2}\right) G\left(\boldsymbol{r}_{1}, \boldsymbol{r}_{2}, t_{1}, t_{2}\right) .
$$

Using $H=H_{0}+H_{1}, G=G_{0}+G_{1}$, and $\Sigma=\Sigma_{0}+\Sigma_{1}$ we obtain

$$
G_{1}=G_{0} *\left(H_{1}+\Sigma_{1}\right) * G_{0} .
$$

The inclusion of $\Sigma_{1}$ is equivalent to accounting for the vertex corrections. This can be seen from Fig. 2 .

The zeroth order in $\boldsymbol{A}$ and $\hat{\Phi}$ Green's functions and self energy reflect the standard disorder broadening. One obtains $\Sigma_{0}^{R}=-i /(2 \tau)$, where $\tau^{-1}=2 \pi n_{\text {imp }} u^{2} \nu$ and $\nu=m^{*} /\left(2 \pi \hbar^{2}\right)$ is the density of states (per spin) without the spin-orbit coupling. The zeroth order (in $\boldsymbol{A}$ and $\hat{\Phi})$ disorder-broadened Green's functions read

$$
G_{0}^{R}=\left(\frac{1}{2}+\frac{1}{2} \frac{\boldsymbol{\eta} \boldsymbol{p}}{p}\right) G_{0}^{R+}+\left(\frac{1}{2}-\frac{1}{2} \frac{\boldsymbol{\eta} \boldsymbol{p}}{p}\right) G_{0}^{R-},
$$

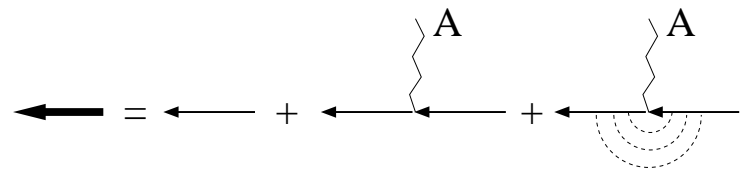

Fig. 2: Diagrammatic representation of Eq. 10. The dashed lines represents the sum of impurity "rainbow" diagrams (Diffuson) with minimum one impurity line.

where $G_{0}^{R \pm}(\boldsymbol{p}, \omega) \equiv\left(\omega-\epsilon^{ \pm}(\boldsymbol{p})+i /(2 \tau)\right)^{-1}$. In equilib$\operatorname{rium} G_{0}^{K}=h(\omega)\left(G_{0}^{R}-G_{0}^{A}\right)$ and $\Sigma_{0}^{K}=h(\omega)\left(\Sigma_{0}^{R}-\Sigma_{0}^{A}\right)$, where $h(\omega) \equiv \tanh \frac{\omega-E_{F}}{2 T}$.

Our goal is to find $G_{1}$ with the self-consistency condition

$$
\Sigma_{1}(\boldsymbol{q}, \omega, \Omega)=\frac{1}{m^{*} \tau} \int \frac{d^{2} p}{(2 \pi)^{2}} G_{1}(\boldsymbol{p}, \boldsymbol{q}, \omega, \Omega) .
$$

Having $G_{1}$ one can calculate any single-particle quantity, i.e., density or current. Thus this approach is equivalent to the Kubo formula with the external vertex (the responding observable) being chosen later.

As usual in the linear response theory the Keldysh component $G_{1}^{K}$ splits into two parts, $G_{1}^{K}=G_{1}^{K, I}+G_{1}^{K, I I}$. Accordingly does the self-energy, $\Sigma_{1}^{K}=\Sigma_{1}^{K, I}+\Sigma_{1}^{K, I I}$. The first part, $G_{1}^{K, I}$, corresponds to the R-A combinations in the Kubo formula, while $G_{1}^{K, I I}$ stands for the R-R and A-A combinations [18]. The spin-charge density matrix is given by $\hat{\rho}=\frac{n}{2}+\boldsymbol{s} \boldsymbol{\sigma}=\int \frac{d \omega}{2 \pi} \int \frac{d^{2} p}{(2 \pi)^{2}}\left[-i G_{1}^{<}\right]=$ $-\frac{i}{2} \int \frac{d \omega}{2 \pi} \int \frac{d^{2} p}{(2 \pi)^{2}}\left(G_{1}^{K}-G_{1}^{R}+G_{1}^{A}\right)=\hat{\rho}^{I}+\hat{\rho}^{I I}$, where $\hat{\rho}^{I}=$ $-\frac{i}{2} \int \frac{d \omega}{2 \pi} \int \frac{d^{2} p}{(2 \pi)^{2}} G_{1}^{K, I}$ and $\hat{\rho}^{I I}$ represents the rest. Here $n(\boldsymbol{q}, \Omega)$ is the charge density while $\boldsymbol{s}(\boldsymbol{q}, \Omega)$ is the spin density. We obtain

$$
\begin{aligned}
& G_{1}^{K, I}(\boldsymbol{p}, \boldsymbol{q}, \omega, \Omega)=G_{0}^{R}(\boldsymbol{p}+\boldsymbol{q} / 2, \omega+\Omega / 2) \cdot \\
& \cdot\left[\{h(\omega+\Omega / 2)-h(\omega-\Omega / 2)\} H_{1}+\Sigma_{1}^{K, I}(\boldsymbol{q}, \omega, \Omega)\right] . \\
& \cdot G_{0}^{A}(\boldsymbol{p}-\boldsymbol{q} / 2, \omega-\Omega / 2) .
\end{aligned}
$$

and $G_{1}^{K, I I}=G_{1}^{R} h(\omega-\Omega / 2)-h(\omega+\Omega / 2) G_{1}^{A}$, where the correction to the retarded Green's function is given by $G_{1}^{R}(\boldsymbol{p}, \boldsymbol{q}, \omega, \Omega)=G_{0}^{R}(\boldsymbol{p}+\boldsymbol{q} / 2, \omega+\Omega / 2)$. $\left[H_{1}(\boldsymbol{p}, \boldsymbol{q}, \omega, \Omega)+\Sigma_{1}^{R}(\boldsymbol{p}, \boldsymbol{q}, \omega, \Omega)\right] \cdot G_{0}^{R}(\boldsymbol{p}-\boldsymbol{q} / 2, \omega-\Omega / 2)$. The dot product is used here to indicate the matrix multiplication.

Our calculations below apply both in the clean (but not "super-clean") regime, $m^{*} \alpha_{\mathrm{R}}^{2}<\tau^{-1}<\Delta_{F}$, and in the dirty regime, $\tau^{-1}>\Delta_{F}$. In the "super-clean" regime, on the other hand, the integration over $\omega$ in the window of width $\Omega$ around the Fermi energy (this window is cut by the function $h(\omega+\Omega / 2)-h(\omega-\Omega / 2)$ in $(13))$ is not smooth and more care has to be taken.

Integrating Eq. (13) over $\boldsymbol{p}$ and $\omega$ we obtain

$$
\frac{(1-I)}{\tau} \hat{\rho}^{I}=i \Omega \nu \tilde{I}\left[\frac{e\{\boldsymbol{v} \boldsymbol{A}\}_{+}}{2 c}-\hat{\Phi}\right]
$$


The functional $\tilde{I}$ is defined as

$$
\begin{aligned}
& \tilde{I}[X(\boldsymbol{p})]= \\
& =\frac{1}{m^{*} \tau} \int \frac{d^{2} p}{(2 \pi)^{2}} G_{0}^{R}\left(\boldsymbol{p}+\boldsymbol{q} / 2, E_{F}+\Omega / 2\right) \cdot X(\boldsymbol{p}) . \\
& \cdot G_{0}^{A}\left(\boldsymbol{p}-\boldsymbol{q} / 2, E_{F}-\Omega / 2\right)
\end{aligned}
$$

while $I$ is a $4 \times 4$ matrix defined by its action on the 4 -vector $\hat{\rho}$ as $I \hat{\rho}=\tilde{I}[\hat{\rho}]$ (we just use the fact that $\hat{\rho}$ is independent of $\boldsymbol{p}$ to represent the functional $\tilde{I}[\hat{\rho}]$ as a product of a $4 \times 4$ matrix $I$ and a vector $\hat{\rho}$ ).

The second contribution to the density, $\hat{\rho}^{I I}$ is given by

$$
\hat{\rho}^{I I}=-\nu \hat{\Phi} \text {. }
$$

Thus the total density follows from

$$
\frac{(1-I)}{\tau}(\hat{\rho}+\nu \hat{\Phi})=i \Omega \nu \tilde{I}\left[\frac{e\{\boldsymbol{v} \boldsymbol{A}\}_{+}}{2 c}-\hat{\Phi}\right] .
$$

We rewrite this equation to have all the terms associated with the perturbing fields on the RHS:

$$
\frac{(1-I)}{\tau} \hat{\rho}=i \Omega \nu \tilde{I}\left[\frac{e\{\boldsymbol{v} \boldsymbol{A}\}_{+}}{2 c}\right]+\frac{[(1-i \Omega \tau) I-1]}{\tau} \nu \hat{\Phi} .
$$

We allow for arbitrary external frequency $\Omega$, including $\Omega>\tau^{-1}$. However, we limit ourselves to the experimentally relevant regime $v_{F}|\boldsymbol{q}| \ll \tau^{-1}$. (Recently the spin and charge response functions to the longitudinal fields were calculated for arbitrary values of $\boldsymbol{q}$ in Ref. [19].) Expanding the RHS of Eq. (18) in powers of $\boldsymbol{q}$ up to the terms linear in $\boldsymbol{q}$ we obtain

$$
\begin{aligned}
& \frac{(1-I)}{\tau} \hat{\rho}=-\nu(e \boldsymbol{E} \cdot \boldsymbol{\eta})\left[\frac{\alpha_{\mathrm{R}} b^{2}}{2 a\left(a^{2}+b^{2}\right)}\right] \\
& -(i \boldsymbol{q} \times e \boldsymbol{E}) \cdot \boldsymbol{\sigma}\left[\frac{b^{2}\left(a^{2}-b^{2}\right)}{8 \pi a\left(a^{2}+b^{2}\right)^{2}}\right] \\
& +\frac{b^{2}}{4 \tau\left(a^{2}+b^{2}\right)} \nu g \mu_{\mathrm{B}}\left(\boldsymbol{B}_{x} \sigma_{x}+\boldsymbol{B}_{y} \sigma_{y}+2 \boldsymbol{B}_{z} \sigma_{z}\right),
\end{aligned}
$$

where $\boldsymbol{E}=i \Omega \boldsymbol{A}-i \boldsymbol{q} \phi$ (we also assumed $\operatorname{div} \boldsymbol{E}=0$ ). We have introduced $a \equiv 1-i \Omega \tau$ and $b \equiv 2 \Delta_{F} \tau$. Note that the magnetic terms $(\propto \boldsymbol{B})$ are already of first order in $\boldsymbol{q}$. The term in the RHS of (19) proportional to $i \boldsymbol{q} \times \boldsymbol{E}=\operatorname{rot} \boldsymbol{E}$ will lead to our central result. The $\boldsymbol{q}$ expansion of the inverse "diffuson" in the LHS of Eq. (18) reads

$$
\begin{aligned}
& \frac{1-I}{\tau} \hat{\rho}= \\
& =\frac{1}{\tau}\left[1-\frac{1}{a}\right] \frac{n}{2}+\frac{1}{\tau}\left[1-\frac{2 a^{2}+b^{2}}{2 a\left(a^{2}+b^{2}\right)}\right]\left(s_{x} \sigma_{x}+s_{y} \sigma_{y}\right) \\
& +\frac{1}{\tau}\left[1-\frac{a}{a^{2}+b^{2}}\right] s_{z} \sigma_{z}-\sigma_{\beta} D_{n m}^{\beta \gamma}\left(i q_{n}\right)\left(i q_{m}\right) \operatorname{Tr}\left[\frac{\hat{\rho} \sigma_{\gamma}}{2}\right] \\
& -\frac{\alpha_{\mathrm{R}} b^{2}}{4 a^{2}\left(a^{2}+b^{2}\right)}\{\boldsymbol{\eta}, i \boldsymbol{q} \hat{\rho}\}_{+}-\frac{i a b v_{F}}{2\left(a^{2}+b^{2}\right)^{2}}[\boldsymbol{\eta}, i \boldsymbol{q} \hat{\rho}]_{-},
\end{aligned}
$$

where $D_{n m}^{\beta \gamma}$ is the diffusion tensor. In the dirty limit, $b \ll$ 1, we obtain $D_{n m}^{\beta \gamma} \approx\left(D / a^{3}\right) \delta_{n m} \delta_{\beta \gamma}$, where $D=v_{F}^{2} \tau / 2$ is the diffusion coefficient. In the clean limit, $b \gg 1$, only the diffusion coefficient in the charge channel is important and we obtain $D_{n m}^{00} \approx\left(D / a^{3}\right) \delta_{n m}$. Eqs. (19) and (20) generalize the results of Refs. $[8,17]$ for arbitrary frequency $\Omega$ and field polarization. Expanding these equations in the dirty limit, $b \ll 1$, and for $\Omega \tau \ll 1$ we obtain the equations of Ref. [17]. The two last terms of Eq. (20) taken in the limit $\Omega \tau \ll 1$ reproduce the spin-charge and spinspin couplings of Ref. [8]. More importantly, we introduce a new driving term in these equations, i.e., the second term in the RHS of Eq. (19) proportional to rot $\boldsymbol{E}$. This term was not appreciated in Refs. $[8,17]$ where only longitudinal driving fields were considered.

We will now use these equations to obtain the spin density. We calculate contributions of zeroth and of first orders in $\boldsymbol{q}, \boldsymbol{s}=\boldsymbol{s}_{0}+\boldsymbol{s}_{1}$. The first order contribution further splits into the orbital and the Zeeman parts, $s_{1}=s_{1}^{\text {orbital }}+s_{1}^{\text {Zeeman }}$.

Zeroth order in $\boldsymbol{q}$. The zeroth order in $\boldsymbol{q}$ corresponds to a homogeneous field. In this case there is no difference between the transverse and the longitudinal cases. Thus we should reproduce known results. For the spin polarization we obtain (cf. Ref. [20])

$$
\boldsymbol{s}_{0}=\alpha_{\mathrm{R}} e E_{x} \tau \nu \boldsymbol{e}_{y} \frac{b^{2}}{b^{2}(2 a-1)+2 a^{2}(a-1)} .
$$

For $\Omega \tau \ll 1$ in the case of strong spin-orbit $(b \gg 1)$ and for $\Omega \ll \Delta_{F}^{2} \tau$ for the case of weak spin-orbit $(b \ll 1)$ this gives $s_{0}(\Omega \rightarrow 0) \approx \alpha_{\mathrm{R}} e E_{x} \tau \nu \boldsymbol{e}_{y}$. In other words this result is obtained when $\Omega \tau_{s} \ll 1$, where $\tau_{s} \equiv \max \left[\tau, 1 /\left(\Delta_{F}^{2} \tau\right)\right]$ is the spin relaxation time. This is the well known result $[2,3]$ meaning that there is an in-plane spin polarization perpendicular to the applied electric field. In the clean limit, $\Delta_{F} \tau \gg 1$, at $\Omega \approx 2 \Delta_{F}$ the polarization (21) shows resonance discussed in Ref. [12].

First (linear) order in $\boldsymbol{q}$. After some algebra we obtain

$$
\begin{aligned}
& \boldsymbol{s}_{1}^{\text {orbital }}= \\
& -\boldsymbol{e}_{z} \frac{i e E_{x} \tau q_{y}}{4 \pi} \frac{b}{\left(b^{2}+a^{2}-a\right)}\left[\frac{b}{2 a}-\Gamma_{0} \frac{a b}{a^{2}+b^{2}}\right] \\
& =\boldsymbol{e}_{z} \frac{m_{e}}{m^{*}} \nu \mu_{\mathrm{B}} B_{z} \frac{b(1-a)}{\left(b^{2}+a^{2}-a\right)}\left[\frac{b}{2 a}-\Gamma_{0} \frac{a b}{a^{2}+b^{2}}\right],
\end{aligned}
$$

where $\Gamma_{0}=\frac{2(a-1)\left(b^{2}+a^{2}\right)}{b^{2}(2 a-1)+2 a^{2}(a-1)}$. We have used $q_{y} E_{x}=$ $-\Omega B_{z} / c$ and $\frac{e}{4 \pi c}=\left(\frac{m_{e}}{m^{*}}\right) \nu \mu_{\mathrm{B}}$, where $\mu_{\mathrm{B}} \equiv e /\left(2 m_{e} c\right)$ and $m_{e}$ is the bare electron mass. Thus we obtain the out-of-plane spin polarization. This is the main result of this work. It follows from the second term in the RHS of Eq. (19). For $\Omega \tau_{s} \ll 1$

$$
\boldsymbol{s}_{1}^{\text {orbital }}(\Omega) \approx-\boldsymbol{e}_{z} \frac{i e E_{x} \tau q_{y}}{8 \pi}=\boldsymbol{e}_{z} \frac{m_{e}}{m^{*}} \nu \mu_{\mathrm{B}} B_{z} \frac{i \Omega \tau}{2} .
$$

The out-of-plane polarization is much smaller than the inplane one given by Eq. (21). Indeed we observe that for 


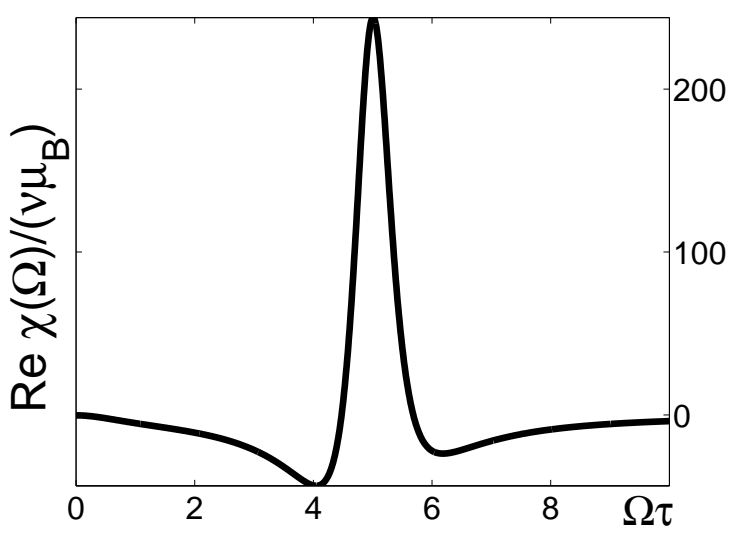

Fig. 3: Real part of the total spin susceptibility $\chi \equiv s_{z} / B_{z}$ for $2 \Delta_{F} \tau=5$. Parameters assumed as in GaAs: $m_{e} / m^{*} \approx 15$, $g \approx-0.44$.

$\Omega \tau_{s} \ll 1$ their ratio is given by $\left|s_{1}^{\text {orbital }}\right| /\left|s_{0}\right| \sim|\boldsymbol{q}| l_{S O}$, where $l_{S O}=\hbar /\left(m^{*} \alpha_{\mathrm{R}}\right)$ is the spin-orbit length. The fact that the result is purely imaginary means that the spin wave is phase shifted by $\pi / 2$ relative to the applied microwave. This is qualitatively consistent with the naive picture of the $z$-polarized spin currents proportional to the electric field (see Fig. 1). However, for quantitative analysis one would need to use the correct continuity equation $-i \Omega s^{z}+i q_{y} j_{y}^{z}=t^{z}$, where $t^{z}$ is the torque density [17].

Near the resonance, $\Omega \sim 2 \Delta_{F}$, in the clean limit, $\Delta_{F} \tau \gg 1$, we obtain

$$
\begin{aligned}
& \boldsymbol{s}_{1}^{\text {orbital }} \approx-\boldsymbol{e}_{z} \frac{m_{e}}{m^{*}} \nu \mu_{\mathrm{B}} B_{z} \times \\
& \times \frac{\Delta_{F}^{2}}{\left(\Omega-2 \Delta_{F}+\frac{i}{2 \tau}\right)\left(\Omega-2 \Delta_{F}+\frac{3 i}{4 \tau}\right)} .
\end{aligned}
$$

Thus we obtain a double pole like in Eq. (6).

Response to the Zeeman term. The Zeeman response follows from the last term in Eq. (19) and is given by

$$
\boldsymbol{s}_{1}^{\text {Zeeman }}=\boldsymbol{e}_{z} \nu \mu_{\mathrm{B}} B_{z} \frac{g}{2} \frac{b^{2}}{b^{2}-(1-a) a} .
$$

In the limit of zero spin-orbit coupling, i.e., $b=0$, but finite $\Omega$ we observe that $s_{1}^{\text {Zeeman }}$ vanishes. The physical meaning of this result is that without the spin-orbit coupling no spin flips are possible and, thus, the spin density cannot react to a dynamical perturbation. The other limiting procedure (first $\Omega \rightarrow 0$, then $b \rightarrow 0$ ) gives the thermodynamic Pauli susceptibility.

Discussion. In the clean limit $\Delta_{F} \tau \gg 1$ the orbital outof plane spin polarization (22) dominates over $s_{z}^{\text {Zeeman }}$ near resonance $\Omega \sim 2 \Delta_{F}$ (although $s_{z}^{\text {Zeeman }}$ is also resonant there). Also in the clean limit the low frequency outof-phase orbital response is dominant, i.e., $\operatorname{Im}\left[s_{1 z}^{\text {orbital }}\right] \gg$ $\operatorname{Im}\left[s_{1 z}^{Z \text { Zeeman }}\right]$ for $\Omega \tau \ll 1$. In the opposite regime, $\Delta_{F} \tau \ll 1$, the ratio of absolute values of the orbital and the Zeeman

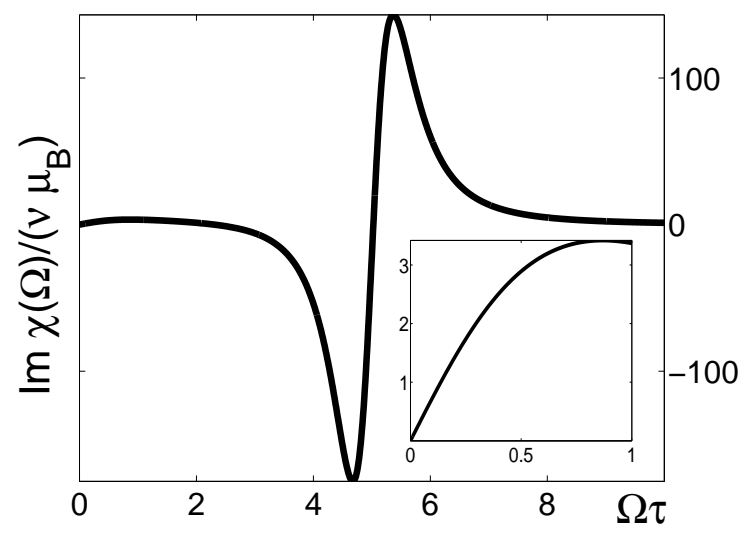

Fig. 4: Imaginary part of the total spin susceptibility $\chi \equiv$ $s_{z} / B_{z}$ for $2 \Delta_{F} \tau=5$. Inset: Blow-up of the imaginary part at $\Omega \tau<1$. Parameters assumed as in GaAs: $m_{e} / m^{*} \approx 15$, $g \approx-0.44$.

contributions becomes independent of $\Delta_{F}$,

$$
\frac{\left|s_{1 z}^{\text {orbital }}\right|}{\left|s_{1 z}^{\text {Zeeman }}\right|} \approx \frac{1}{g} \frac{m_{e}}{m^{*}} \frac{\Omega \tau}{\sqrt{1+\Omega^{2} \tau^{2}}} .
$$

If the prefactor $(1 / g)\left(m_{e} / m^{*}\right)$ is large (as it happens, e.g., in GaAs) the orbital contribution dominates at high enough frequencies.

It is convenient to measure the susceptibility in units of $\nu \mu_{\mathrm{B}}$. In Figs. 3 and 4 the resonant behavior at high frequency is shown. For plotting we choose the clean regime, $2 \Delta_{F} \tau=5$. The orbital Fano-like contribution is clearly pronounced. The linear in frequency dependence of the imaginary part at $\Omega \tau<1$ (shown in the inset in Fig. 4) corresponds to Eq. (23).

For numerical estimates we take the parameters of the 2DEG in GaAs/AlGaAs heterostructures from Ref. [21]. With $m^{*} \sim 0.067 m_{e} \sim 0.7 \cdot 10^{-31} \mathrm{~kg}$ we obtain the density of states $\nu=m^{*} /\left(2 \pi \hbar^{2}\right) \sim 10^{36} \mathrm{~J}^{-1} \mathrm{~m}^{-2}$. With $\mu_{\mathrm{B}} \sim 10^{-23} \mathrm{~J} / \mathrm{T}$ we obtain $\nu \mu_{\mathrm{B}} \sim 10^{13} \mathrm{~T}^{-1} \mathrm{~m}^{-2}=$ $10^{5} \mathrm{G}^{-1} \mathrm{~cm}^{-2}$. With the mobility $\mu=e \tau / \mathrm{m}^{*} \sim 25 \mathrm{~m}^{2} / \mathrm{Vs}$ and the electron density $n_{0} \sim 6 \cdot 10^{15} \mathrm{~m}^{-2}$ we obtain $\tau \sim 10^{-11} \mathrm{~s}, v_{F} \sim \sqrt{n_{0} /\left(m^{*} \nu\right)} \sim 3 \cdot 10^{5} \mathrm{~m} / \mathrm{s}$, and the mean free path $l=\tau v_{F} \sim 3 \mu \mathrm{m}$. The reported value for the spin-orbit length $l_{S O} \sim 4 \mu \mathrm{m}$. Thus we are on the border between the Dyakonov-Perel (dirty) regime and the strong spin-orbit regime so that $\Delta_{F} \tau=l / l_{S O} \sim 1$.

The oscillating transverse electric field needed to generate the bulk spin accumulation described here can be created inside a microwave wave guide or a strip-line resonator. Assuming for the estimate a microwave field with $B_{z} \sim 1$ Gauss, the spin polarization on resonance is about $2 \cdot 10^{7} \mathrm{~cm}^{-2}$. Such polarization should be measurable with the magneto-optical spectroscopic techniques $[22,23]$. Alternatively, the transverse (circulating) electric field could be generated with an AC magnet.

We acknowledge discussions with M. Freeman, A. Shekhter, M. Pletyukhov, A. G. Yashenkin, and S. 
Crooker. This work was supported by the DOE and by the CFN of the DFG.

\section{REFERENCES}

[1] M. I. Dyakonov and V. I. Perel, Sov. Phys. JETP 33, 1053 (1971).

[2] A. G. Aronov and Yu. B. Lyanda-Geller, JETP Letters 50, 431 (1989).

[3] V. M. Edelstein, Solid State Commun. 73, 233 (1990).

[4] J. Sinova, D. Culcer, Q. Niu, N. A. Sinitsyn, T. Jungwirth, and A. H. MacDonald, Phys. Rev. Lett. 92, 126603 (2004).

[5] S. Murakami, N. Nagaosa, and S. C. Zhang, Science 301, 1348 (2003).

[6] S. Murakami, Adv. Solid State Phys. 45, 197 (2005).

[7] J. Inoue, G. E. W. Bauer, and Laurens W. Molenkamp, Phys. Rev. B 70, 041303 (2004).

[8] E. G. Mishchenko, A. V. Shytov, and B. I. Halperin, Phys. Rev. Lett. 93, 226602 (2004).

[9] O. V. Dimitrova, Phys. Rev. B 71, 245327 (2005).

[10] O. Chalaev and D. Loss, Phys. Rev. B 71, 245318 (2005).

[11] S. I. Erlingsson, J. Schliemann, and D. Loss, Phys. Rev. $B$ 71, 035319 (2005).

[12] A. Shekhter, M. Khodas, and A. M. Finkel'stein, Phys. Rev. B 71, 165329 (2005).

[13] E. I. Rashba, J. of Superconductivity: Incorp. Nov. Magn. 18, 137 (2005).

[14] D. Culcer, Y. Yao, and Q. Niu, Phys. Rev. B 72, 085110 (2005).

[15] C. P. Slichter, Principles of Magnetic Resonance, Springer 3d edition (1996).

[16] M. Duckheim and D. Loss, Nature Physics 2, 195 (2006).

[17] A. A. Burkov, A. S. Núñez, and A. H. MacDonald, Phys. Rev. B 70, 155308 (2004).

[18] J. Rammer and H. Smith, Rev. Mod. Phys. 58, 323 (1986).

[19] M. Pletyukhov, cond-mat/0611523 (2006).

[20] R. Raimondi, C. Gorini, P. Schwab, and M. Dzierzawa, Phys. Rev. B 74, 035340 (2006).

[21] D. M. Zumbühl, J. B. Miller, C. M. Marcus, K. Campman, and A. C. Gossard, Phys. Rev. Lett. 89, 276803 (2002).

[22] S. A. Crooker, D. D. Awschalom, J. J. Baumberg, F. Flack, and N. Samarth, Phys. Rev. B 56, 7574 (1997).

[23] Y. K. Kato, R. C. Myers, A. C. Gossard, and D. D. Awschalom, Science 306, 1910 (2004). 